\title{
Safety of stereotactic ablative body radiation for ultracentral stage I non-small cell lung cancer
}

\author{
Abraham Jing-Ching Wu \\ Memorial Sloan Kettering Cancer Center, New York, NY, USA \\ Correspondence to: Abraham Jing-Ching Wu. Memorial Sloan Kettering Cancer Center, New York, NY, USA. Email: wua@mskcc.org.
}

\begin{abstract}
Recent data suggests that "ultra-central" tumors, generally defined as those abutting the proximal airways, are at particularly high risk for severe complications when treated with stereotactic ablative body radiation (SABR). However, this association has not been consistently demonstrated across reports, possibly due to small numbers, varying definitions of "ultra-central", and the lack of prospective data. New evidence suggests that exposure to VEGF-inhibiting agents may potentiate SABR toxicity and may partially explain the disproportionately high incidence of fatal complications in some reports. Efforts are underway to identify dose-volume limits that can predict complications involving central structures such as the proximal airways, heart, esophagus, and great vessels. The optimal dose for ultra-central SABR has not been determined, though there is a trend towards using more highly fractionated regimens. Further research into the safety of SABR for ultra-central tumors is needed, given the lack of other effective local therapy options for this clinical scenario.
\end{abstract}

Keywords: Stereotactic ablative body radiation (SABR); stereotactic body radiation therapy (SBRT); non-small cell lung cancer (NSCLC); ultra-central; central

Submitted Jul 04, 2019. Accepted for publication Aug 13, 2019.

doi: $10.21037 /$ tlcr.2019.08.08

View this article at: http://dx.doi.org/10.21037/tlcr.2019.08.08

Stereotactic ablative body radiation (SABR) is now a wellestablished treatment option for early-stage non-small cell lung cancer (NSCLC) and small lung metastases given its high rates of local control, relatively low toxicity, and short treatment times compared to conventionally fractionated radiation. Seminal prospective trials for both primary NSCLC (1) and lung metastases (2) demonstrated the efficacy and safety of SABR and propelled its rapid adoption and establishment as a first-line non-surgical treatment for small lung tumors. However, the early prospective experience at Indiana University with lung SABR in unselected patients revealed an excessive risk of significant toxicity if the tumor was near the central airways (3). This led to the definition and rapid adoption of the "no-fly-zone", defined as a $2 \mathrm{~cm}$ radius around the proximal bronchial tree (PBT). Subsequent clinical practice and prospective studies in lung SABR made a clear distinction between tumors in "central" and "peripheral" zones.

However, a clear explanation for increased toxicity from
SABR in the central lung zone has not yet been elucidated, and the "no-fly-zone" remains an empirically derived and abstract concept, rather than a robust model for risk stratification. It is worth noting that a subsequent publication of the Indiana University experience no longer demonstrated a significant difference in toxicity between peripheral and central tumors with longer follow-up (4). This underscored the lingering questions about the lack of clear validation for the "no-fly-zone" as a framework for predicting toxicity after SABR, and leaves open the possibility that other definitions or risk factors may more accurately guide treatment (5). Compounding this uncertainty is the fact that the original definition of "central" tumors has not been consistently applied. The RTOG 0813 trial, in particular, adopted a more permissive definition that also included tumors adjacent to the mediastinal or pericardial pleura even if they were outside the no-fly-zone (6).

Despite these differences and ongoing debates, the Indiana University data essentially led to the 
contraindication of high-dose three-fraction SABR regimens (18 Gy per fraction or higher) for central lesions. Since reverting to conventional radiation treatment for central lesions was not a very appealing option either, many subsequent experiences and reports used lower SABR doses with more fractions, most commonly five. One early and large retrospective series from Memorial Sloan Kettering Cancer Center (MSKCC) indicated that with these riskadapted fractionations (most commonly, 45 Gy in five fractions), relatively high rates of local control could be achieved without excessive toxicity (7). A systematic review of twenty publications on SABR for central lung tumors, published in 2013, concluded that local control rates on the order $85 \%$ could be achieved, with rates of severe toxicity under $10 \%$ (8).

The most authoritative data on the safety of five-fraction SABR for central NSCLC has now been presented in the form of the RTOG 0813 trial, a prospective multiinstitutional trial that treated 120 patients with five-fraction SABR and successfully achieved a maximum tolerated dose of $12 \mathrm{~Gy}$ per fraction. This dose level was associated with a $7.2 \%$ probability of dose-limiting toxicities (6). The recent official publication of RTOG 0813 therefore validates the safety of five-fraction SABR for central lung tumors, a scheme that had already become standard practice at many leading SABR centers.

However, the definition of "central" remains quite broad: a tumor that barely intersects the no-fly-zone is still considered "central", even though the bulk of the tumor is peripheral. And as noted above, a tumor outside the nofly-zone but abutting the mediastinal envelope would still be considered "central" according to RTOG 0813, even though there is little reason to think that a small tumor barely abutting the descending aorta, for example, should be at especially high risk for SABR-related complications. Therefore, even after the publication of RTOG 0813 there remains significant clinical uncertainty whether doses of 50-60 Gy in five fractions are suitable for all central tumors regardless of their relationship or proximity to central structures.

Concern over especially severe complications even with risk-adapted SABR regimens for central tumors was heightened in 2012 with the high-profile publication of a case report describing fatal hemoptysis (after 50 Gy in 5 fractions) to a tumor in close proximity to the right mainstem bronchus (9). In 2015, a group from Stanford published a report including seven patients with "ultracentral tumors", which they defined as GTV directly abutting a major airway (trachea, mainstem bronchus, or lobar bronchus) (10). Patients received 50 Gy in 4-5 fractions and no significant increase in toxicity was observed in the ultra-central patients. However, as the authors acknowledged, the number of ultra-central patients was small.

At MSKCC, we analyzed a larger group of central SABR patients to determine whether the degree of proximity to the major airways was a predictor of severe necrosis. In a series of 108 patients, we observed that grade $3+$ toxicity was significantly more likely when the tumor was $\leq 1 \mathrm{~cm}$ from the PBT, compared to tumors $1-2 \mathrm{~cm}$ from the PBT (11). This series, published in 2016, also included 18 ultra-central patients (GTV abutting PBT), of whom four experienced SABR-related death. This relatively high proportion of death was notable, as was the fact that no cases of SABRrelated death were observed in the remainder of the "central" patients.

Since then, several other series of ultracentral SABR have been reported, with varying conclusions about whether such patients should be considered a high-risk subset of central tumors requiring a unique approach. The VU University Medical Center reported a relatively large series (47 patients) of ultra-central tumors, but they used a more liberal definition of PTV, rather than GTV, overlapping the trachea or main bronchi. Fatal pulmonary hemorrhage was observed in $15 \%$ of patients, which the authors considered a concerningly high rate (12). In contrast, a Canadian series compared 46 ultracentral (defined as GTV abutting PBT) with 61 central patients and found no significant difference in grade $\geq 3$ toxicity between the groups (13).

MSKCC has since published the largest series of ultracentral tumors treated with SABR to date, a cohort of 88 patients (14). The most notable finding is the statistically significant correlation between antiangiogenic agent exposure (such as bevacizumab) and the incidence of fatal pulmonary hemorrhage. Four of six patients who experienced SABR-related death had exposure to such agents, and led us to the conclusion that the combination of SABR and antiangiogenic therapy produces synergistic toxicity in ultracentral patients and therefore should be avoided. An association between antiangiogenic agents and SABR-induced toxicity has previously been described in abdominal sites (15).

Ideally, questions about the safety of SABR in ultracentral tumors should be answered in the prospective setting. The RTOG 0813 trial described above included a relatively small number of ultracentral patients and was not specifically 
designed to compare ultracentral $v s$. central patients. A Canadian trial, SUNSET (ClinicalTrials.gov identifier: NCT03306680), has now been activated with the aim of determining the maximum tolerated SABR dose in ultracentral patients, though it uses the more liberal definition of PTV rather than GTV overlapping PBT.

Whether ablative radiotherapy doses can safely be given to ultracentral tumors therefore remains an unanswered question. It also is a clinically important one. Not only can primary lung cancers arise in an ultracentral location, but metastases as well. Because of rapidly increasing interest in radical local therapy for oligometastatic disease, determining the optimal local treatment for ultracentral tumors is a crucial one, as this is not an uncommon presentation. Ultracentral lung metastases often are not technically feasible to be surgically resected (at least, not without large operations such as bilobectomy or pneumonectomy) or undergo radiofrequency ablation, leaving radiotherapy as the only remaining local therapy option. While conventionally fractioned radiation or low-dose SABR (such as 30 Gy in 5 fractions) could be given, the lower biologically effective dose means that durable local control is unlikely, potentially defeating the purpose of comprehensive radical local therapy.

Besides prospective trials, an important component of answering safety questions around ultracentral SABR is the effort to identify precise dose-volume limits for the proximal airways and other mediastinal structures, such as the heart, esophagus, and great vessels. The RTOG 0618 trial specified comprehensive dose-volume constraints for normal structures, and further reports focusing on dosimetric details and analyses should be valuable, and are eagerly awaited. The VUMC group has published an analysis of dosimetric predictors for high-grade toxicity in central SABR patients, derived from detailed study of bronchial structures in 195 patients (16). More such data is needed, though the challenge will then be to synthesize data from different series and institutions and derive generalizable and consensus predictors of toxicity. This is far from assured, given the very heterogeneous nature of ultracentral tumor presentations and definitions, and the increasing variety of SABR fractionation schemes being employed at various centers. There also remains the distinct possibility that tumors directly abutting the PBT or other important mediastinal structures increase the risk of SABR through direct physical invasion, in which case normal tissue dose-volume metrics alone would not entirely predict the risk of severe toxicity.

At our center we have moved towards 8- and 15-fraction schemes (both to a total dose of 60 Gy) when deploying SABR for ultracentral tumors, both of which have precedent in the literature. We favor the 8-fraction regimen, which was popularized by VUMC, for smaller tumors abutting the PBT (17). The 15-fraction regimen is derived from a Canadian Phase II trial, and we favor this scheme for tumors with a greater degree of encasement of the proximal airways, or an otherwise greater interface with sensitive mediastinal structures (18). It should be noted that the biologically effective dose for this 15 -fraction regimen is lower as well.

In conclusion, the safety of SABR for ultracentral NSCLC remains uncertain, despite an increasing number of retrospective analyses, and the publication of the RTOG 0813 trial. Although there remains relatively little published data specifically on ultra-central tumors, and not all extant reports identified higher toxicity, it seems prudent to regard ultra-central tumors as an especially high-risk subset warranting a distinct approach from other central tumors. For example, we would not consider 60 Gy in 5 fractions to be advisable for ultracentral tumors in general, despite the results of RTOG 0813, but would favor more highly fractionated, and possibly lower-BED, schemes. Due to the association with fatal pulmonary hemorrhage, it is strongly discouraged to use SABR for ultracentral tumors in close proximity ( $\leq 30$ days) to exposure to antiangiogenic agents.

Whether risk-adapted fractionation (e.g., using more than 5 fractions to deliver an ablative dose) can effectively mitigate severe toxicity remains to be seen. It is also hoped that robust dose-volume limits for normal structures will be validated, to guide the appropriate application of SABR in this setting. Undoubtedly, more robust data and guidelines are needed. For now, the question of SABR for ultracentral tumors (both primary NSCLC and oligometases) will remain one of the most delicate clinical judgments a radiation oncologist must make. It requires us to consider uncertain and serious risks, which nevertheless may be outweighed by the potentially grave consequences of tumor progression in a location that frequently cannot be addressed with any other local treatment modality.

\section{Acknowledgments}

None.

\section{Footnote}

Conflicts of Interest: The author has no conflicts of interest to 
declare.

Ethical Statement: The author is accountable for all aspects of the work in ensuring that questions related to the accuracy or integrity of any part of the work are appropriately investigated and resolved.

\section{References}

1. Timmerman R, Paulus R, Galvin J, et al. Stereotactic body radiation therapy for inoperable early stage lung cancer. JAMA 2010;303:1070-6.

2. Rusthoven KE, Kavanagh BD, Burri SH, et al. Multiinstitutional phase I/II trial of stereotactic body radiation therapy for lung metastases. J Clin Oncol 2009;27:1579-84.

3. Timmerman R, McGarry R, Yiannoutsos C, et al. Excessive toxicity when treating central tumors in a phase II study of stereotactic body radiation therapy for medically inoperable early-stage lung cancer. J Clin Oncol 2006;24:4833-9.

4. Fakiris AJ, McGarry RC, Yiannoutsos CT, et al. Stereotactic body radiation therapy for early-stage non-small-cell lung carcinoma: four-year results of a prospective phase II study. Int J Radiat Oncol Biol Phys 2009;75:677-82.

5. Oskan F. The Quality of Toxicity Reporting and the Story of the Lung SBRT "No-Fly Zone". Int J Radiat Oncol Biol Phys 2015;92:514-5.

6. Bezjak A, Paulus R, Gaspar LE, et al. Safety and Efficacy of a Five-Fraction Stereotactic Body Radiotherapy Schedule for Centrally Located Non-Small-Cell Lung Cancer: NRG Oncology/RTOG 0813 Trial. J Clin Oncol 2019;37:1316-25.

7. Modh A, Rimner A, Williams E, et al. Local control and toxicity in a large cohort of central lung tumors treated with stereotactic body radiation therapy. Int J Radiat Oncol Biol Phys 2014;90:1168-76.

8. Senthi S, Haasbeek CJ, Slotman BJ, et al. Outcomes of stereotactic ablative radiotherapy for central lung tumours: a systematic review. Radiother Oncol 2013;106:276-82.

Cite this article as: Wu AJ. Safety of stereotactic ablative body radiation for ultracentral stage I non-small cell lung cancer. Transl Lung Cancer Res 2019;8(Suppl 2):S135-S138. doi: 10.21037/tlcr.2019.08.08
9. Corradetti MN, Haas AR, Rengan R. Central-airway necrosis after stereotactic body-radiation therapy. N Engl J Med 2012;366:2327-9.

10. Chaudhuri AA, Tang C, Binkley MS, et al. Stereotactic ablative radiotherapy (SABR) for treatment of central and ultra-central lung tumors. Lung Cancer 2015;89:50-6.

11. Haseltine JM, Rimner A, Gelblum DY, et al. Fatal complications after stereotactic body radiation therapy for central lung tumors abutting the proximal bronchial tree. Pract Radiat Oncol 2016;6:e27-33.

12. Tekatli H, Haasbeek N, Dahele M, et al. Outcomes of Hypofractionated High-Dose Radiotherapy in Poor-Risk Patients with "Ultracentral" Non-Small Cell Lung Cancer. J Thorac Oncol 2016;11:1081-9.

13. Chang JH, Poon I, Erler D, et al. The safety and effectiveness of stereotactic body radiotherapy for central versus ultracentral lung tumors. Radiother Oncol 2018;129:277-83.

14. Wang C, Rimner A, Gelblum DY, et al. Analysis of Toxic Effects With Antiangiogenic Agents Plus Stereotactic Body Radiation in Ultracentral Lung Tumors. JAMA Oncol 2019;5:737-9.

15. Barney BM, Markovic SN, Laack NN, et al. Increased bowel toxicity in patients treated with a vascular endothelial growth factor inhibitor (VEGFI) after stereotactic body radiation therapy (SBRT). Int J Radiat Oncol Biol Phys 2013;87:73-80.

16. Tekatli H, Duijm M, Oomen-de Hoop E, et al. Normal Tissue Complication Probability Modeling of Pulmonary Toxicity After Stereotactic and Hypofractionated Radiation Therapy for Central Lung Tumors. Int J Radiat Oncol Biol Phys 2018;100:738-47.

17. Haasbeek CJ, Lagerwaard FJ, Slotman BJ, et al. Outcomes of stereotactic ablative radiotherapy for centrally located early-stage lung cancer. J Thorac Oncol 2011;6:2036-43.

18. Cheung P, Faria S, Ahmed S, et al. Phase II study of accelerated hypofractionated three-dimensional conformal radiotherapy for stage T1-3 N0 M0 non-small cell lung cancer: NCIC CTG BR.25. J Natl Cancer Inst 2014. doi: 10.1093/jnci/dju164. 